\title{
Nonstructural Protein of Parvoviruses B19 and Minute Virus of Mice Controls Transcription
}

\author{
CHRISTIAN DOERIG, $\dagger$ BERNHARD HIRT, JEAN-PHILIPPE ANTONIETTI, AND PETER BEARD* \\ Department of Virology, Swiss Institute for Experimental Cancer Research, 1066 Epalinges, Switzerland
}

Received 24 July 1989/Accepted 29 September 1989

\begin{abstract}
The genome of the human parvovirus B19 contains a transcriptional promoter (BP06) at map position 6 , upstream from the nonstructural protein genes. By cotransfecting HeLa cells with this promoter cloned before the chloramphenicol acetyltransferase (CAT) gene together with a plasmid containing almost the whole B19 genome, we showed that BP06 is transactivated by a B19 gene product. The transactivating viral protein was identified as the nonstructural protein NS-1. NS-1 synthesized in a wheat germ extract specifically stimulates transcription from BP06 in vitro. NS-1 of the minute virus of mice (MVM) activates the analogous MVM promoter, MP04. NS-1, therefore, has a positive feedback effect on the activity of its own promoter. Moreover, NS-1 of MVM activates the human BP06. We have identified, in the genome of B19, a second transcriptional promoter activity at map position 44 , before the capsid protein genes. This promoter, BP44, was identified by cloning fragments of B19 DNA upstream of the CAT gene, transfecting the DNA into HeLa cells, and measuring CAT expression. The strength of the BP44 promoter is similar to that of the capsid gene promoter, MP39, of MVM. In (nonpermissive) HeLa cells, the BP44 promoter is not activated by NS-1. Thus, the BP06 promoter apparently does not determine the tissue specificity of B19 virus but BP44 could do so.
\end{abstract}

Autonomous parvoviruses show a strong tropism towards dividing cells because of their requirement for a cellular factor expressed during the $S$ phase of the cell cycle. Several of these viruses are extremely tissue specific, able to grow only in cells at a particular stage of differentiation (for reviews, see references 5,39 , and 44 ).

B19 virus is the first human autonomous parvovirus described (8). It is the causal agent of erythema infectiosum, or fifth disease (1). B19 virus also provokes aplastic crises in anemics $(31,36)$, a consequence of the fact that the virus grows lytically in erythroid progenitor cells at a precisely defined stage of differentiation (24). Until now, no cell line permissive for B19 virus growth has been found. The virus is able to replicate to some extent in cultures of freshly isolated human bone marrow cells, although permissive cells represent only a minor portion of these cultures (27). Cotmore and Tattersall (11) have cloned most of the genome of B19 virus from a viremic serum, which allowed the determination of the viral DNA sequence (38). The genomic organization of B19 virus resembles that of other autonomous parvoviruses in terms of number and location of open reading frames (ORFs): there are two large nonoverlapping ORFs, together occupying a large part of the genome. In addition, two small ORFs are found in the middle and near the right end of the viral DNA $(10,38)$. We and others $(6,17,26)$ showed previously that a transcriptional promoter (BP06) is present at the left end of the genome of B19 virus; this promoter is active in vitro as well as in vivo.

In comparison with B19 virus, the autonomous parvoviruses, minute virus of mice (MVM) and the closely related $\mathrm{H}-1$ virus, have been well studied (for a review, see reference 43). Two strains of MVM have been isolated: MVMi, infecting lymphocytes, and MVMp, infecting fibroblasts (2, 42). The DNA sequences of H-1 (30) and of both strains of $\operatorname{MVM}(3,4,37)$ have been determined. The genomes of these

\footnotetext{
* Corresponding author.

$\dagger$ Present address: Department of Microbiology and Immunology, University of Colorado, Health Sciences Center, Denver, CO 80262.
}

viruses contain two overlapping transcription units (29): one promoter (MP04) located at 4 map units, directs transcription of the genes of the two nonstructural proteins, whose coding region is situated in the left part of the genome; the promoter of the second unit (MP39) is located at 39 map units and is responsible for the transcription of the capsid protein genes situated on the right part of the genome $(12,20$, 34). The MP04 promoter is active earlier in the lytic cycle than is MP39 (8). Rhode (33) has reported that the nonstructural protein, NS-1, of $\mathrm{H}-1$ virus is involved in the transactivation of the capsid gene promoter and has identified a cis-acting element present in this promoter which responds to NS-1 (35). We have previously shown, by cotransfection experiments, that in MVMi the nonstructural protein NS-1 (hereafter called MNS-1) stimulates in trans the MP39 promoter (17).

In the work reported here, we investigated the effect of the nonstructural proteins NS-1 of B19 virus and of MVMi on the activity of their own promoters, BP06 and MP04. Unexpectedly, we found a positive feedback effect. Until now, there has been no report of a late promoter upstream of the capsid protein genes of B19 virus (26). This is surprising, since other parvoviruses contain such a late promoter (5). By cloning fragments of B19 viral DNA into plasmid vectors containing the chloramphenicol acetyltransferase (CAT) gene, we found evidence for a promoter at map position 44 just before the capsid protein genes of B19 virus.

\section{MATERIALS AND METHODS}

T4 DNA polymerase, T4 DNA ligase, restriction enzymes, SP6 RNA polymerase, RNase-free DNase, and RNasin were purchased from Boehringer $\mathrm{GmbH}$, Mannheim, Federal Republic of Germany, and were used according to Maniatis et al. (22) or the protocol of the supplier.

Plasmid construction. The construction of pMP39-CAT, pEMBL/i0099 (2), pEMBL/i0052, pMNS-1 ${ }^{\mathrm{m}}$, pMNS-2 ${ }^{\mathrm{m}}$, pMNS-2 ${ }^{\mathrm{m}}$ cap $^{\mathrm{m}}$ (17), pEMBL/B19, and pBP06-CAT (16) has been described elsewhere. 


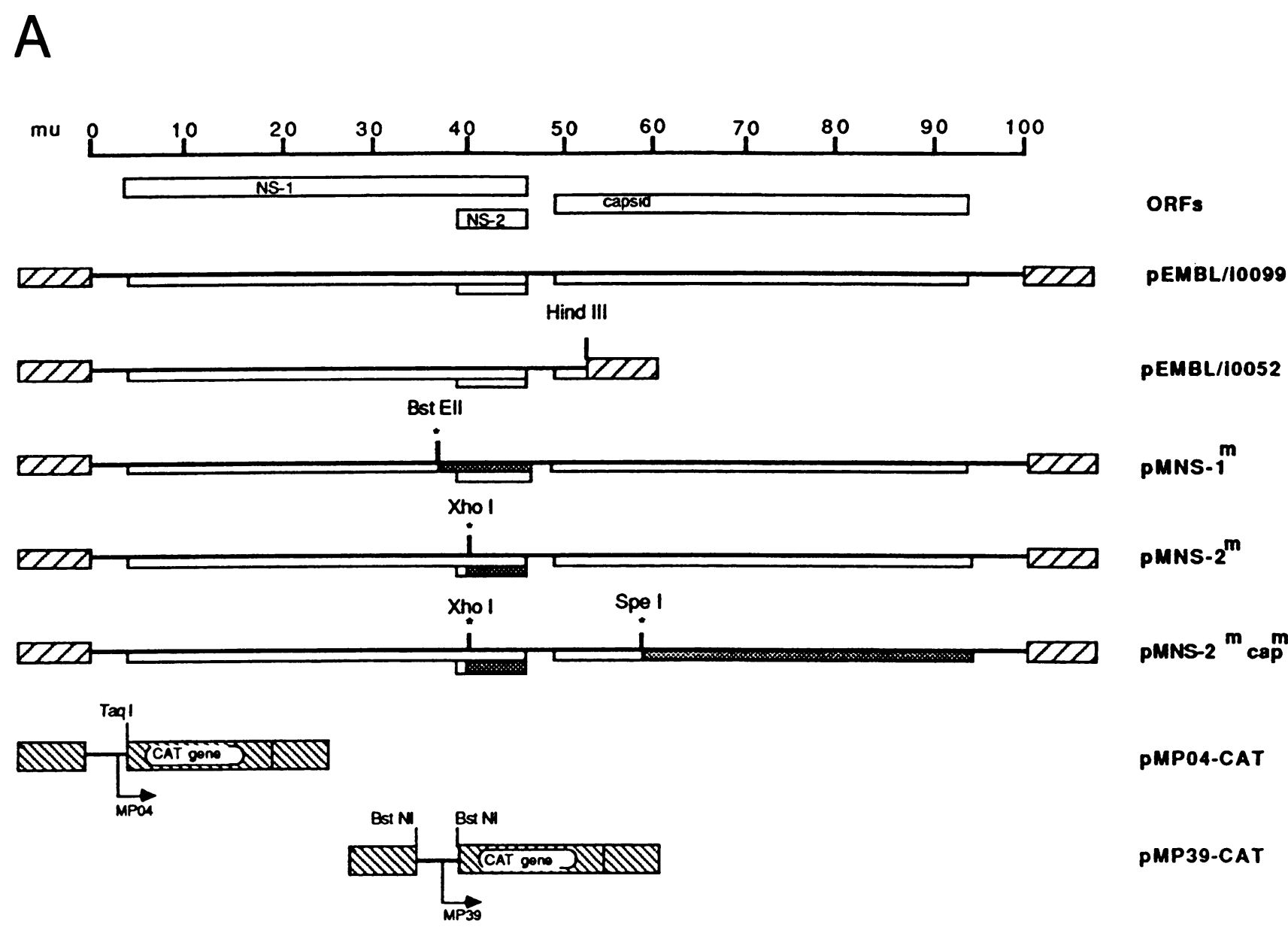

FIG. 1. Plasmids used in this work. (A) Plasmids containing MVM DNA. The ORF labeled NS-2 is the ORF which is unique to NS-2 and so can be used to specifically mutate NS-2. The asterisks show mutated sites, and cross-hatched boxes show ORFs no longer read manually (17). (B) Plasmids containing B19 DNA. Symbols: ${ }_{\downarrow}$, TATA-box-related sequence; $\mathbb{Q}$, pSV0-CAT; $\mathbb{Z}$, pEMBL.

Construction of pMP04-CAT. The TaqI restriction fragment containing the MP04 promoter was isolated from the clone pEMBL/i0099; one TaqI site is in the polylinker, and the other one is at position 228 , so that the fragment was about 240 base pairs long. This fragment was blunt ended with T4 DNA polymerase, ligated to HindIII linkers, and inserted into the HindIII cloning site of pSV0-CAT. Both orientations were obtained as determined by digestion with Hinfl (site at position 225).

Construction of pSP6-BNS-1. We inserted the B19 virus restriction fragments RsaI-HindIII (nucleotides [nt] 333 to 595) and HindIII-PstI (nt 595 to 3145) into a SmaI- and PstI-cut pSP65 vector in a single trimolecular ligation reaction (see Fig. 3); the RsaI and SmaI blunt ends were ligated together, allowing the viral HindIII-PstI fragment to insert in its expected position. Recombinant plasmids were checked by digestion with restriction enzymes and by transcription with SP6 RNA polymerase of the plasmid linearized with different restriction enzymes.

Construction of pBP44-CAT. We isolated the SmaITth111I fragment (nt 2073 to 2277) from pEMBL/B19, made it blunt with T4 DNA polymerase, ligated it to 8-mer HindIII linkers, and inserted it into the HindIII cloning site of the plasmid pSV0-CAT. Both orientations were obtained, as determined by digestion of the recombinant plasmid with
AvalI (position 2075). The other B19-CAT plasmids (Fig. 1) were constructed by similar methods.

Construction of pB19-cap ${ }^{m}$. This clone was constructed by digestion of $\mathrm{pEMBL} / \mathrm{B} 19$ with PstI, followed by isolation of the larger fragment (containing the vector [14] and the larger viral Pst I fragment, from nt 1 to 3145 ) and subsequent religation.

Transfection of HeLa and EL-4 cells and CAT assays. HeLa or EL-4 cells were plated on 6 -cm petri dishes $\left(6 \times 10^{5}\right.$ cells per plate) $24 \mathrm{~h}$ before transfection. The DNA (purified by a CsCl-ethidium bromide gradient) was transfected into $\mathrm{HeLa}$ cells by the calcium phosphate method of Graham and Van der Eb (19) and into EL-4 cells by the DEAE-dextran method of Sompayrac and Danna (40). At $48 \mathrm{~h}$ after transfection, the cells were washed, harvested, suspended in 100 $\mu \mathrm{l}$ of $250 \mathrm{mM}$ Tris hydrochloride, $\mathrm{pH} 7.5$, and lysed by three cycles of freezing and thawing. The cell debris was removed by centrifugation, and the supernatant was heated for $5 \mathrm{~min}$ at $65^{\circ} \mathrm{C}$. This step greatly enhances the CAT activity of a given extract, presumably by inactivating a inhibitor or a deacetylating activity present in the extract. The assay mixture (18) contained $20 \mu$ l of cell extract, $19 \mu$ l of $250 \mathrm{mM}$ Tris hydrochloride $(\mathrm{pH} 7.5), 1 \mu \mathrm{l}$ of ${ }^{14} \mathrm{C}$-labeled chloramphenicol $(0.2 \mathrm{mCi} / \mathrm{ml}, 54 \mathrm{mCi} / \mathrm{mmol}$; Amersham International, United Kingdom) and $5 \mu \mathrm{l}$ of $44 \mathrm{mM}$ acetylcoenzyme 
B
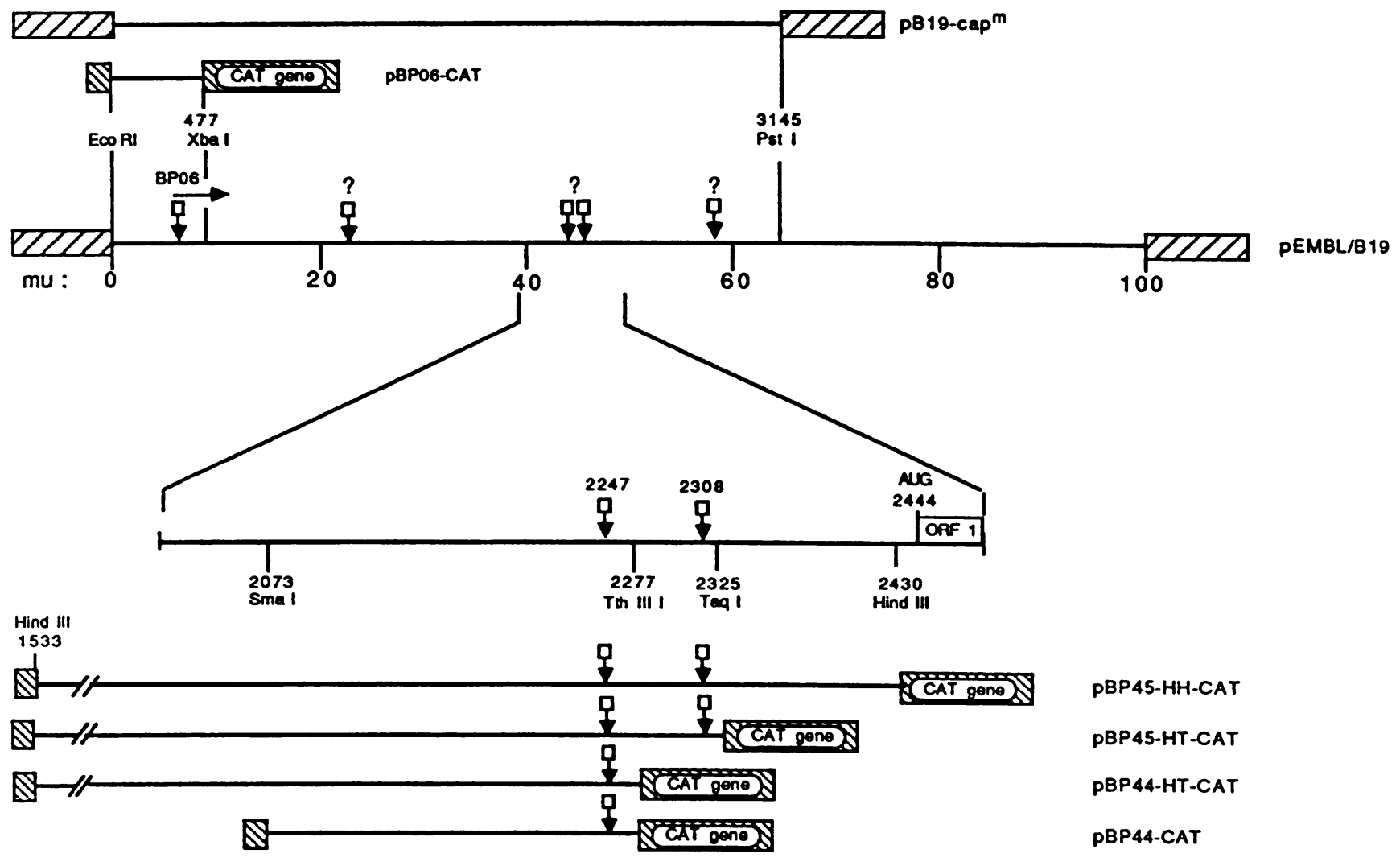

PBP45-HH-CAT

PBP45-HT-CAT

PBP44-HT-CAT

PBP44-CAT

FIG. 1-Continued.

A. The reaction was carried out at $37^{\circ} \mathrm{C}$ for $14 \mathrm{~h}$ and stopped by addition of $0.5 \mathrm{ml}$ of ethylacetate, which was also used to extract the chloramphenicol and its acetylated derivatives. (Control experiments showed that the 14-h incubation gave qualitatively the same results as a 1-h incubation, but with greater sensitivity.) The organic layer was lyophilized, and the chloramphenicol was taken up in $30 \mu$ l of ethylacetate, spotted on silica gel thin-layer plates, and chromatographed with chloroform-methanol (95:1). The plate was then dried and exposed to film (Kodak X-AR5) for autoradiography.

Transcription in vitro with SP6 RNA polymerase and translation in vitro with wheat germ extract. We have slightly modified the method of Struhl and Hope (41), which derives from that of Melton et al. $(21,23)$. One microgram of template DNA (SP6-BNS-1 linearized with PstI) was transcribed in a reaction $(25 \mu \mathrm{l})$ containing $40 \mathrm{mM}$ Tris hydrochloride ( $\mathrm{pH} \mathrm{8.0),} 6 \mathrm{mM} \mathrm{MgCl}_{2}, 2 \mathrm{mM}$ spermidine, $10 \mathrm{mM}$ $\mathrm{NaCl}, 100 \mu \mathrm{g}$ of bovine serum albumin, $1 \mathrm{mM}$ dithiothreitol, $1 \mathrm{U}$ of RNasin per $\mu \mathrm{l}, 500 \mu \mathrm{M}$ ATP, CTP, and UTP, $500 \mu \mathrm{M}$ diGTP (3'OH-G-ppp-G-3'OH) (Pharmacia, Inc.), $50 \mu \mathrm{M}$ GTP, and 200 to $500 \mathrm{U}$ of SP6 RNA polymerase per ml. The diGTP is an analog of the cap structure, and its presence during the transcription improves the ability of the RNA to be translated (41). After 45 to $60 \mathrm{~min}$ at $40^{\circ} \mathrm{C}$, we added 450 $\mu M$ GTP and let the reaction go on for another 30 to $45 \mathrm{~min}$. The DNA template was then digested by addition of RNasefree DNase I $(400 \mathrm{U} / \mathrm{ml})$ during $20 \mathrm{~min}$ at $37^{\circ} \mathrm{C}$. After phenol extraction, the RNA (about $10 \mu \mathrm{g}$ according to approximate quantitation by ethidium bromide staining) was precipitated with ethanol.

The RNA was dissolved in water, and one-third of it (about $3 \mu \mathrm{g}$ ) was added to a $100-\mu \mathrm{l}$ translation reaction containing $50 \mu \mathrm{l}$ of wheat germ extract (Amersham), $100 \mu \mathrm{M}$ amino acids minus Met, $1 \mu \mathrm{Ci}$ of $\left[{ }^{35} \mathrm{~S}\right] \mathrm{Met}(>800 \mathrm{Ci} / \mathrm{mmol}, 15$ $\mathrm{mCi} / \mathrm{ml}$ ) per $\mu \mathrm{l} 500 \mathrm{U}$ of RNasin per $\mathrm{ml}$, and $72 \mathrm{mM}$ $\mathrm{CH}_{3} \mathrm{COOK}\left(72 \mathrm{mM}\right.$ is the optimal $\mathrm{K}^{+}$concentration we determined for a maximal incorporation of $\left[{ }^{35} \mathrm{~S}\right] \mathrm{Met}$ into protein; this optimal concentration depends on the mRNA used as a template). As a negative control, we did the same reaction but added water without RNA. Incorporated radioactivity was measured by incubation of a portion at $37^{\circ} \mathrm{C}$ in $50 \mathrm{mM} \mathrm{NaOH}$ (to hydrolyze amino-acyl-tRNA) followed by trichloroacetic acid precipitation, washing on glass fiber filters, and counting in a scintillation counter.

NS-1 was immunoprecipitated from in vitro translation mixtures by using rabbit antiserum prepared against a bacterially produced trpE-NS-1 fusion protein (F. Beutler and C. Doerig, unpublished data). Antiserum and the solution containing NS-1 were mixed at $4^{\circ} \mathrm{C}$ for $2 \mathrm{~h}$, one-half volume of protein A-Sepharose beads (Pharmacia) was added, and the incubation was continued for $1 \mathrm{~h}$. The beads were collected by centrifugation, and washed three times with a solution of $10 \mathrm{mM}$ Tris hydrochloride ( $\mathrm{pH} \mathrm{8),} 1 \mathrm{mM}$ EDTA, and $100 \mathrm{mM} \mathrm{NaCl}$, and then suspended in sample buffer for polyacrylamide gel electrophoresis.

Transcription in vitro with HeLa cell extracts. HeLa nu- 


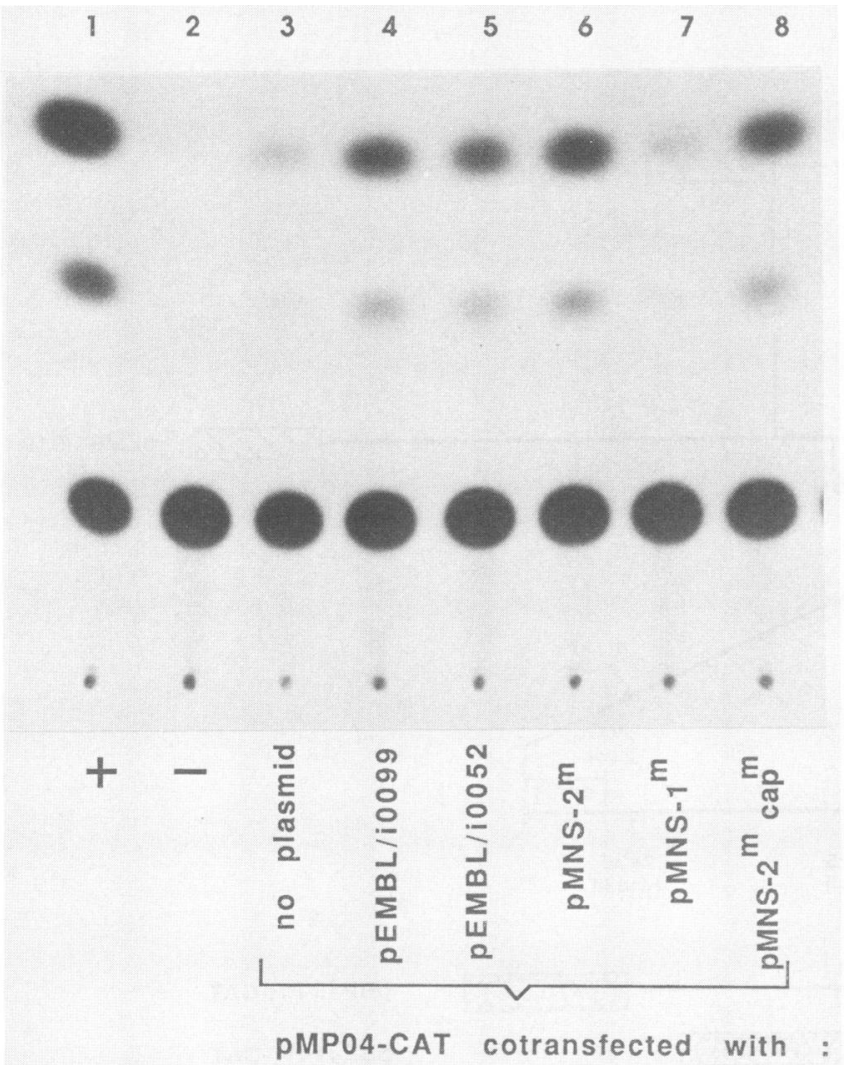

FIG. 2. Transactivation of the MP04 promoter by NS-1 in transfected EL-4 cells. EL-4 cells were transfected with $0.5 \mu \mathrm{g}$ of pMP04-CAT and $0.5 \mu \mathrm{g}$ of the indicated plasmids per $6-\mathrm{cm}$ petri dish by the DEAE-dextran method (17). At $48 \mathrm{~h}$ posttransfection, we prepared cell extracts and measured the CAT activity, using ${ }^{14} \mathrm{C}$ labeled chloramphenicol as a substrate (17). The acetylated and the unacetylated forms of chloramphenicol were separated by thin-layer chromatography and localized by autoradiography. The controls were cells transfected with $5 \mu \mathrm{g}$ of pRSV-CAT (lane 1) and cells transfected with $5 \mu \mathrm{g}$ of promoterless pSV0-CAT (lane 2) (17). Other lanes indicate pMP04-CAT cotransfected with no plasmid (lane 3), pEMBL/i0099 (lane 4), pEMBL/i0052 (lane 5), pMNS-2 ${ }^{\mathrm{m}}$ (lane 6), pMNS- $1^{\mathrm{m}}$ (lane 7), and pMNS-2 ${ }^{\mathrm{m}} \mathrm{cap}^{\mathrm{m}}$ (lane 8).

clear extracts were made by the procedure of Dignam et al. (15). The transcription reaction $(50 \mu \mathrm{l})$ contained 0.2 to $1 \mu \mathrm{g}$ of DNA template, $200 \mu \mathrm{M}$ ATP, GTP, and CTP, $20 \mu \mathrm{M}$ UTP, $10 \mu \mathrm{Ci}$ of $\left[{ }^{32} \mathrm{P}\right] \mathrm{UTP}(400 \mathrm{Ci} / \mathrm{mmol}), 5 \mathrm{mM}$ creatine phosphate, $0.1 \mu \mathrm{g}$ of poly(dIC), and $25 \mu \mathrm{l}$ of HeLa nuclear extract; portions from in vitro translation reactions were added as described in the text. The reaction was carried out at $30^{\circ} \mathrm{C}$ for $1 \mathrm{~h}$, and the nucleic acids were then purified by digestion with pronase $(0.1 \mu \mathrm{g} / \mathrm{ml})$ for $20 \mathrm{~min}$ at $37^{\circ} \mathrm{C}$ in the presence of $1 \%$ sodium dodecyl sulfate extraction with phenol, addition of $10 \mu \mathrm{g}$ of carrier tRNA, and ethanol precipitation. They were redissolved in water and denatured by glyoxal $\left(15 \mathrm{~min}\right.$ at $50^{\circ} \mathrm{C}$ in the presence of 2 volumes of $75 \%$ dimethyl sulfoxide, $15 \mathrm{mM} \mathrm{NaPO}_{4}$ [pH 6.9], $0.15 \%$ sodium dodecyl sulfate, and $7 \%$ glyoxal). Electrophoresis was carried out in a $1.8 \%$ agarose gel in $10 \mathrm{mM} \mathrm{NaPO}_{4}, \mathrm{pH}$ 6.9. The gel was then dried and exposed to a Kodak X-AR5 film for autoradiography.

\section{RESULTS}

Transactivation of the early promoter MP04 of MVM by NS-1 in transfected EL-4 cells. The transactivation of the capsid gene promoter by NS-1 of autonomous parvoviruses is well established $(16,31,32)$. We asked whether the NS-1 protein of MVMi (that is MNS-1) also controls the activity of its own promoter, MP04.

We constructed a reporter plasmid (pMP04-CAT) in which the CAT gene was under the control of the MP04 promoter. When we tested the ability of this plasmid to express CAT upon transfection into EL-4 cells, we observed that only the clone in which the viral fragment was in the right orientation relative to the CAT gene (i.e., with the TaqI site at position 228 proximal to the CAT gene) expressed CAT (see Fig. 2) and not the clone in which the viral fragment was inserted in the opposite orientation (not shown). Thus, the MP04 promoter was functional in our reporter plasmid. We then tested whether a clone (pEMBL/i0099) $(2,17)$ containing the whole MVMi genome (except a part of the $5^{\prime}$ palindrome) expressed a product that would activate in trans the production of CAT from pMP04-CAT. If a viral gene product expressed from the genomic clone is able to activate transcription from a viral promoter controlling the transcription of the CAT gene, we should observe increased CAT activity in cells containing both plasmids compared with cells containing only the CAT construct. EL-4 cells were transfected with a mixture of pMP04-CAT and pEMBL/i0099, and the CAT activity was measured in cell extracts. It is clear from Fig. 2 that the CAT activity was higher in cells transfected with both plasmids (lane 4) than in cells transfected with pMP04CAT alone (lane 3). There was about five times more acetylated chloramphenicol in lane 4 of Fig. 2 than in lane 3, as determined by scintillation counting.

To determine which of the viral proteins was responsible for this transactivation, we performed similar experiments in which the plasmid cotransfected with pMP04-CAT was from a series of clones able to express subsets of viral proteins. The structure of these plasmids and how they were made has already been described (17). When the plasmid cotransfected with pMP04-CAT was pEMBL/i0052, which lacks most of the capsid protein-coding sequences, the CAT activity (Fig. 2 , lane 5) was again higher than in cells transfected with pMP04-CAT alone (Fig. 2, lane 3). This indicated that a nonstructural protein transactivates the MP04 promoter. To determine which nonstructural protein, we cotransfected pMP04-CAT with either pMNS- $1^{\mathrm{m}}$ or $\mathrm{pMNS}-2^{\mathrm{m}}$. These plasmids are unable to express intact either MNS-1 or MNS-2, respectively (17). We observed that $\mathrm{pMNS}-2^{\mathrm{m}}$ was able to transactivate CAT expression from pMP04-CAT to a level equivalent to that produced by cotransfection of pMP04-CAT and pEMBL/i0099 (Fig. 2, lanes 4 and 6). pMNS- $1^{\mathrm{m}}$, on the other hand, had no effect on the expression of CAT from pMP04-CAT (Fig. 2, lane 7). Thus, NS-1 seems to be necessary for the transactivation of MP04. The clone pMNS $-2^{\mathrm{m}}$ which expresses NS-1 (and the domain of NS-2 which is shared with NS-1) transactivates MP04 as efficiently as does the whole MVMi genome. We noted that the CAT activity was somewhat lower when transactivation was by pEMBL/i0052 (Fig. 2, lane 5) rather than by the whole viral genome (Fig. 2, lane 4). Two interpretations are possible: either the capsid proteins are needed in conjunction with MNS-1 for a maximum level of transactivation or the lack of the polyadenylation site for the MNS-1 mRNA in pEMBL/i0052 leads to a reduced expression of MNS-1. To distinguish these two possibilities, we measured transactivation by the plasmid pMNS $-2^{\mathrm{m}}$ cap $^{\mathrm{m}}$, which contains the polyadenylation site and is able to express MNS-1 but neither MNS-2 nor the capsid proteins (17). We observed (Fig. 2, lane 8) that this plasmid stimulated, to the same 


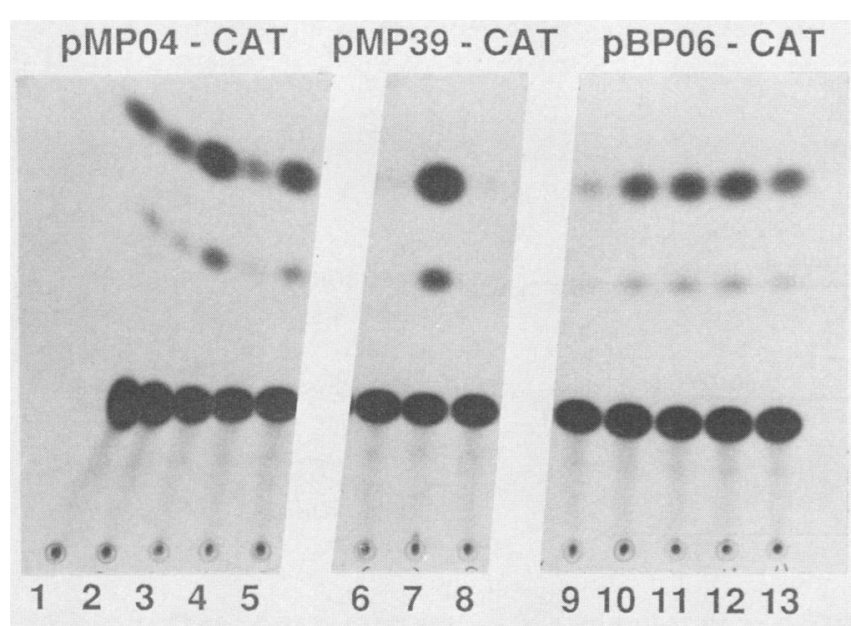

FIG. 3. Transactivation of B19 virus and MVM promoters by virus-coded proteins in transfected HeLa cells. CAT assays were as described in the legend to Fig. 2. The promoter-containing plasmids tested were pMP04-CAT $(0.5 \mu \mathrm{g})$ (lanes 1 through 5), pMP39-CAT (1 $\mu \mathrm{g})$ (lanes 6 through 8), and pBP06-CAT $(0.5 \mu \mathrm{g})$ (lanes 9 through 13). The transactivating plasmids are indicated: none in lanes 1,6 , and 9; pMNS-2 ${ }^{\mathrm{m}}$ cap $^{\mathrm{m}}, 0.5 \mu \mathrm{g}$ in lanes 2 and 12 and $1 \mu \mathrm{g}$ in lanes 3, 7 , and 13; pB19 $\mathrm{cap}^{\mathrm{m}}, 0.5 \mu \mathrm{g}$ in lane 4 and $1 \mu \mathrm{g}$ in lanes 5 and 8; pEMBL/B19, $0.5 \mu \mathrm{g}$ in lane 10 and $1 \mu \mathrm{g}$ in lane 11 .

extent as pEMBL/i0099, the production of CAT from pMP04-CAT. This result argues against an involvement of the capsid proteins in transactivation of the MP04 promoter in this experiment. Our interpretation of these results is that NS-1 has a positive feedback effect on the MP04 promoter, in addition to its known ability to transactivate the MP39 promoter.

The BP06 promoter is transactivated by a B19-encoded gene product in transfected HeLa cells. Since expression from both viral promoters of MVMi is activated in trans by MNS-1, we asked whether transactivation of expression from the promoter BP06 $(6,16)$ of the human parvovirus B19 by a B19 virus-encoded gene product occurs. We constructed a plasmid, pBP06-CAT (16), with the BP06 promoter linked to the bacterial CAT gene. We performed cotransfection experiments in which the plasmid pBP06-CAT was transfected into HeLa cells together with the plasmid pEMBL/B19. This latter clone (16) derives from the plasmid pYT103 described by Cotmore and Tattersall (11) and contains almost the complete genome of B19 virus (minus a part of the terminal hairpin structures).

Figure 3, lanes 9 through 11 show that the BP06 promoter is activated in trans by a B19 gene product; this transactivation is similar in strength to that of MP04 by MNS-1 (lanes 1 through 3). By analogy to MVM, the protein responsible for the transactivation of the BP06 promoter might be expected to be BNS-1. To test this hypothesis and to check in a more direct way the effect of BNS-1 on the activity of the BP06 promoter, we synthesized BNS-1 in vitro and tested the ability of this protein to modulate transcription from the BP06 promoter in vitro.

In vitro synthesis of BNS-1. We obtained BNS-1 in vitro by translating in a cell extract the RNA synthesized by the SP6 RNA polymerase from the plasmid pSP6-BNS-1 (Fig. 4A). Transcripts produced by SP6 RNA polymerase with this plasmid used as a template are shown in Fig. 4B. We linearized the plasmid template with PstI and used the runoff transcripts synthesized (containing the whole BNS-1 coding region) as a template for cell-free translation in a wheat germ extract (Fig. 4C). The protein produced had the expected apparent molecular mass as deduced from the DNA sequence (38), about 72 kilodaltons (Fig. 4C, lane 4). The strong band at 68 kilodaltons, corresponding presumably to premature termination of translation, has also been detected in in vitro translation experiments using mRNA extracted from B19-infected bone marrow cells $(17,28)$. The bands of lower molecular mass were probably also products of premature translation termination, since they were absent in reaction mixtures containing no exogenous mRNA (data not shown). The full-length protein and the lower-molecularmass bands were immunoprecipitable with an anti-BNS-1 antiserum raised against a fusion protein containing the carboxy-terminal part of BNS-1 (Fig. 4C, lanes 2 and 3) but not with a preimmune serum (Fig. 4C, lane 1). We conclude from these results that the protein we synthesized in vitro was BNS-1.

BNS-1 synthesized in vitro stimulates transcription from the BP06 promoter in vitro. To determine whether the BNS-1 protein synthesized in vitro had an effect on the activity of B19 promoters, we performed in vitro transcription assays in HeLa cell nuclear extracts, using restriction fragments of B19 DNA as templates. Portions of translation reaction mixtures with either BNS-1 mRNA or no exogenous mRNA (and therefore containing or not the BNS-1 protein) were added to transcription reactions, and the yield of runoff transcripts were compared. The presence or absence of BNS-1 was the only difference between these two transcription reactions, since the same volume of in vitro translation mixture was added to each. Figure 5, lanes 1 and 2, show the runoff transcripts generated from the B19 virus PstI A fragment in the absence (lane 1) or in the presence (lane 2) of the in vitro-synthesized BNS-1 protein. In the presence of BNS-1, the increase in the quantity of the transcript of 2.8 kilobases originating at the BP06 promoter (16) was clear. The intensity of the nonspecific bands did not vary significantly between the two lanes. (The nonspecific bands were possibly due to either end labeling of endogenous RNA molecules present in the extract or to transcripts originating in the vector DNA). To make sure the increase in the intensity of the 2.8-kilobase band was not due to the labeling of the pSP6-BNS-1-derived mRNA which was used as a template for the synthesis of BNS-1 (and which has a size near that of the runoff transcript), we repeated the experiment, using the BamHI A fragment as a template for in vitro transcription. This fragment yields a transcript of about 3.4 kilobases when RNA synthesis is initiated at the BP06 promoter (16). As can be seen in lanes 3 and 4 of Fig. 5, the addition of a portion from a translation reaction mixture containing BNS-1 also increased the yield of transcripts from the BamHI A fragment. Therefore, the increase in labeled RNA we detected in the presence of BNS-1 reflects a stimulation of transcription of the restriction fragment template. When, in parallel reactions, we tested the effect of BNS-1 on the activity of the simian virus 40 early promoter (Fig. 5, lanes 5 and 6), we observed no increase but rather a slight decrease in the quantity of simian virus 40 transcripts (about 2 kilobases) in the presence of BNS-1. The effect we observed on the BP06 promoter was, therefore, not due to a nonspecific enhancement of the transcriptional activity in the HeLa extract by BNS-1. We conclude from these results that BNS-1 is able to transactivate the BP06 promoter in vitro.

The NS-1 proteins of both B19 virus and MVMi are able to activate both BP06 and MP04. Can the NS-1 protein of one 

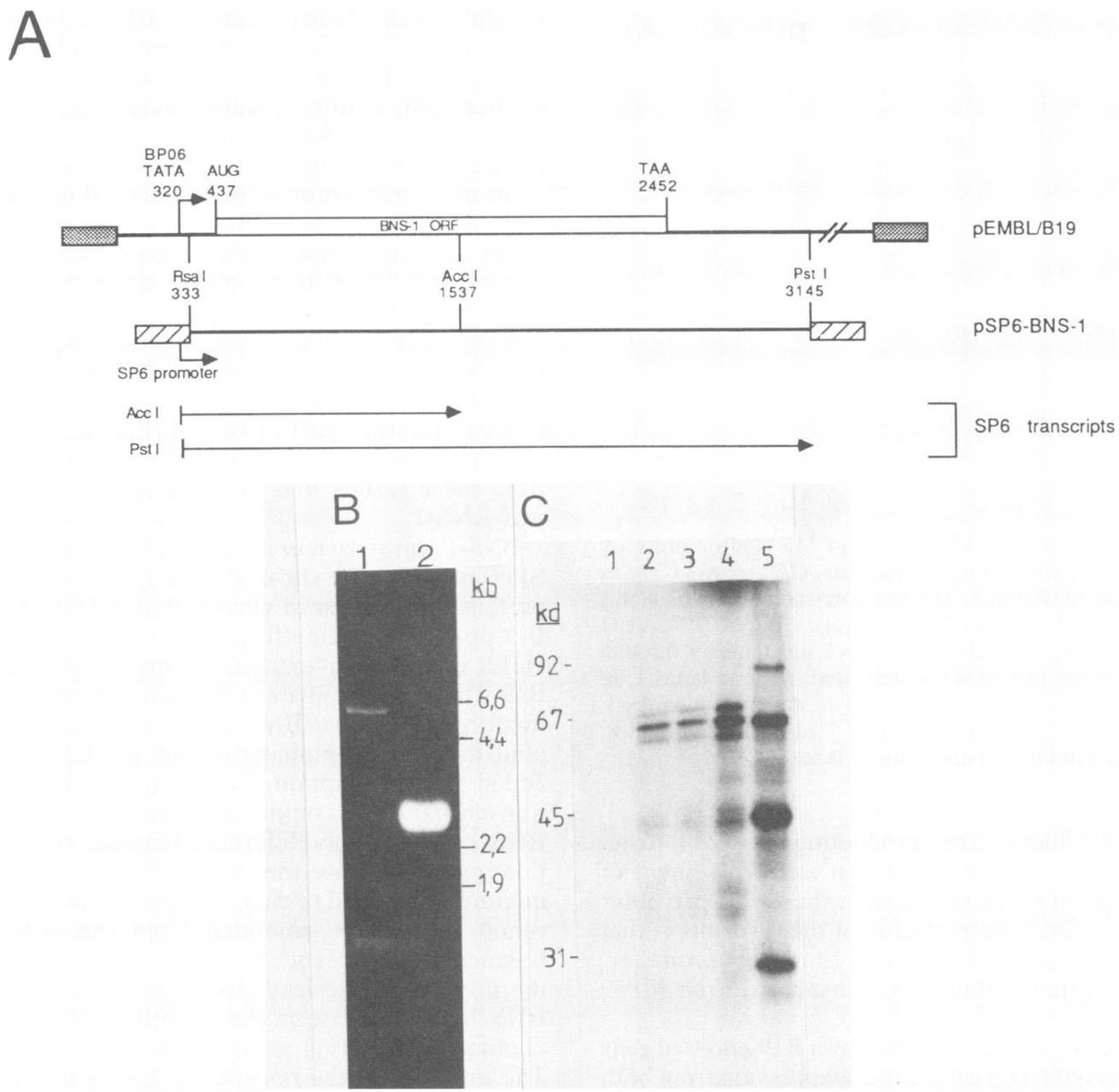

FIG. 4. In vitro synthesis of the NS-1 protein of B19 virus. (A) Structure of plasmid pSP6-BNS-1. The runoff RNAs expected on transcription of the plasmid linearized with AccI or PstI by SP6 RNA polymerase are shown. (B) Runoff RNAs made by SP6 RNA polymerase. The pSP6-BNS-1 template was linearized with AccI (lane 1) or PstI (lane 2). RNAs were reacted with glyoxal (described in Materials and Methods), electrophoresed in an agarose gel, and stained with ethidium bromide. Sizes of bands (in kilobases) are indicated on the right. (C) ${ }^{35}$ S-labeled proteins made in vitro from SP6 RNA polymerase-synthesized in BNS-1 mRNA. Proteins were made in a wheat germ extract and immunoprecipitated by preimmune serum (lane 1) or anti-BNS-1 sera (lanes 2 and 3 ) or the translation reaction mixture was analyzed without immunoprecipitation (lane 4). Molecular size markers are indicated in lane 5, and sizes are given in kilodaltons on the left. Proteins were electrophoresed in a polyacrylamide gel and detected by autoradiography.

parvovirus activate transcription from the other? To answer this question, we first performed cotransfections of HeLa cells by using pBP06-CAT and pMNS-2 ${ }^{\mathrm{m}} \mathrm{cap}^{\mathrm{m}}$; this latter plasmid is a modified MVMi clone able to express only MNS-1 (17). In Fig. 3, lanes 9 through 13 show that MNS-1 is able to activate expression from the BP06 promoter with the same efficiency as BNS-1. We also tested the symmetrical combination and cotransfected pMP04-CAT together with $\mathrm{pB} 19 \mathrm{cap}^{\mathrm{m}}$, a clone containing the left part of B19 virus genome, from the left end to the PstI site at position 3145 (Fig. 1). The result (Fig. 3, lanes 1 through 5) shows that B19 virus nonstructural protein activates expression from the MP04 promoter but to a lesser extent than MNS-1 does. Such a cross-transactivation was not observed with the capsid gene promoter MP39: this promoter was activated by pMNS-2 ${ }^{\mathrm{m}}$ cap $^{\mathrm{m}}$ but not by pB19 cap $^{\mathrm{m}}$ (Fig. 3, lanes 6 through 8).

Promoter activity at 44 map units on the genome of the B19 virus in transfected HeLa cells. The existence in B19 virus of a promoter (BP06) near the left end of the genome is a feature shared by all the autonomous parvoviruses studied so far. The other parvoviruses, but not B19 (26), have been shown to have a second promoter located near the middle of the genome (at 39 map units in the case of MVM) controlling the transcription of the capsid protein genes. Since the genome of B19 virus contains TATA-box-related sequences at map positions 44 and $45(38)$, we decided to determine whether or not a DNA fragment carrying one or both of these TATA boxes was able to promote transcription in vivo. For this purpose, we constructed a series of plasmids in which the gene for the bacterial enzyme CAT was placed downstream of these TATA boxes (Fig. 1), transfected them into HeLa cells, and measured the CAT activity in the transfected cells. The cells transfected with plasmids containing both TATA elements did not produce any CAT activity above the background level (data not shown); on the other hand, the plasmid pBP44-CAT, containing the viral fragment SmaI-Tth111I (nt 2071 through 2247), induced the expression of CAT (Fig. 6, lane 6). The same fragment inserted in the opposite orientation relative to the CAT gene 


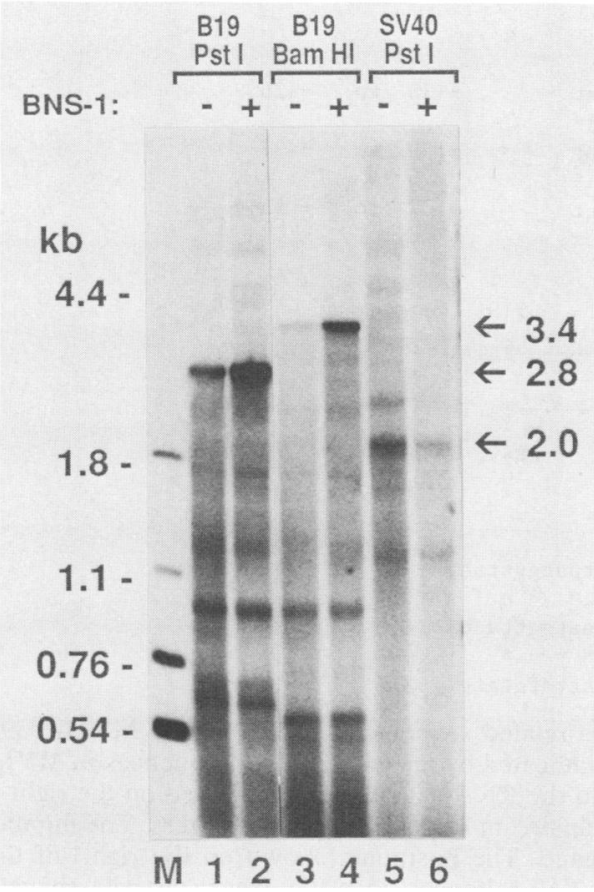

FIG. 5. Effect of adding BNS-1 protein synthesized in vitro on transcription. In vitro transcription reactions contained as template pEMBL/B19 digested by PstI (lanes 1 and 2) or by BamHI (lanes 3 and 4) or simian virus 40 DNA digested by PstI (lanes 5 and 6). BamHI cuts B19 viral DNA at position 3896, and Pst I cuts at positions 3145 and 3860 . The transcription reactions $(50 \mu \mathrm{l})$ included $3 \mu \mathrm{l}$ of in vitro translation mix either with (lanes 2, 4, and 6) or without (lanes 1, 3, and 5) pSP6-BNS-1 RNA. Sizes of bands (in kilobases) are indicated on the left; the runoff transcript bands of interest are indicated by the arrows on the right. Size markers were $H i n f I-d i g e s t e d$ simian virus 40 DNA and HindIII-digested $\lambda$ DNA.

did not lead to the production of CAT (Fig. 6, lane 1). By transfecting various amounts of DNA, the strength of the promoter activity in pBP44-CAT was compared with that in pMP39-CAT (a plasmid in which the CAT gene is under the control of the MP39 promoter of MVMi [17]). The two promoters had very similar activities (Fig. 6, compare lanes 2 through 4 with lanes 6 through 8 , respectively).

The basal activity of the BP44 promoter in pBP44-CAT was not increased when the HeLa cells were cotransfected with $\mathrm{pEMBL} / \mathrm{B} 19$ containing almost all the B19 genome (Fig. 6, lane 9). The BP44 promoter in plasmid pBP44HT-CAT which contains a longer stretch of upstream sequence (Fig. 1) was likewise not transactivated (data not shown). The MP39 promoter of MVM, on the other hand, was transactivated by cotransfection with the equivalent MVM clone, pEMBL/i0099 (Fig. 6, lane 5; 17). MP39 was not activated by NS-1 of B19 virus nor was BP44 activated by MNS-1 (Fig. 3, lane 8; results not shown).

\section{DISCUSSION}

Positive feedback regulation of the early promoter of autonomous parvoviruses. The conclusion that the NS-1 protein of autonomous parvoviruses has a positive feedback effect on the activity of its own promoter is based on several independent lines of evidence. Firstly, cells transfected with this promoter of B19 virus or MVM linked to the CAT gene produced more CAT activity when cotransfected with a

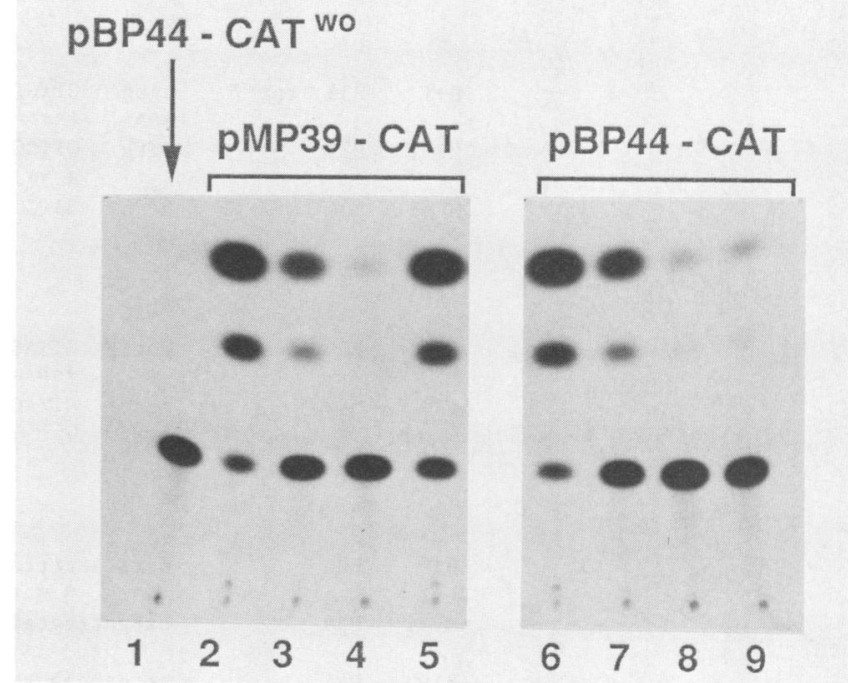

FIG. 6. The activities of promoters MP39 and MP44 in HeLa cells. The transfected plasmids (and amounts) were the following: $\mathrm{pBP}_{44-C A T}{ }^{\text {wo }}$ (15 $\mu \mathrm{g}$ [lane 1]; this plasmid has the promoter sequence inserted in the wrong orientation); pMP39-CAT $(10 \mu \mathrm{g}$. [lane 2]; $5 \mu \mathrm{g}$ [lane 3] and $1 \mu \mathrm{g}$ [lanes 4 and 5]); pEMBL/10099 $(1 \mu \mathrm{g})$ was cotransfected in lane 5; pBP44-CAT (10 $\mu \mathrm{g}$ [lane 6], $5 \mu \mathrm{g}$ [lane 7], and $1 \mu \mathrm{g}$ [lanes 8 and 9]); and pEMBL/B19 (1 $\mu \mathrm{g})$ was also cotransfected in lane 9. CAT assays were as described in the legend for Fig. 2.

plasmid expressing NS-1. Regulation of CAT expression could occur at various levels (43). Secondly, the amount of mRNA transcribed from the MP04 promoter is lower in transfected cells containing a mutated NS-1 protein than in cells containing the wild-type NS-1 (17). Thirdly, NS-1 of B19 virus synthesized in vitro activates transcription from BP06 in vitro. The same result has been obtained with MVM NS-1 and the promoter MP04 (unpublished results).

Rhode and Richard (35) characterized a cis-acting element in the P38 promoter of $\mathrm{H}-1$ virus which responds to NS-1 ( $\mathrm{H}-1$ is a virus closely related to MVM). They reported that the minimal sequence required for transactivation is a sequence of 13 base pairs, named tar, located between positions -138 and -126 relative to the start site of the capsid gene mRNA. The MP39 promoter of MVMi has an almost identical sequence between positions 1868 and 1880 (that is, from -139 to -127 relative to the cap site of the capsid mRNA; see Fig. 7). This is not surprising, since the viruses are related. A DNA sequence related to the central core portion of the tar element, TGGTTGGT, is present unexpectedly often throughout the MVM genome (7). The function of this sequence is not yet clear. Nevertheless, what is interesting in view of our finding that MP04 is also activated by NS-1 is that upstream of the MP04 promoter, between map positions 96 and 105 ( -107 to -95 from the nonstructural protein mRNA cap site), is a sequence with high homology to a longer stretch of the tar present in the MP39 promoter (Fig. 7). This sequence, CNTGGTTGGTNAA, occurs at only these two places in the MVMi genome. The function of these sequences in MVMi has not yet been tested, but, although it is not proven, we suspect that they share the same functional properties as the H-1 P38 tar.

Contradictory results have been reported previously for the effect of NS proteins on early promoter function. It has been stated (35) that NS-1 of the $\mathrm{H}-1$ parvovirus inhibits rather than stimulates the activity of the P04 promoter. Since 
A.

\begin{tabular}{|c|c|c|c|c|c|c|c|c|}
\hline $\mathrm{H}-1$ & P38 & tar & $\begin{array}{l}\operatorname{tgtg} \\
\star \star \star \star \star\end{array}$ & 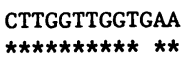 & $\begin{array}{l}\text { gaat } \\
\star \star \star \star \star\end{array}$ & -138 & to & -126 \\
\hline MVMi & P39 & & $\operatorname{tg} t g$ & $\begin{array}{l}\text { CTTGGTTGGTAAA } \\
\star \star \star \star \star \star \star \star \star \star\end{array}$ & $\begin{array}{r}\text { gaat } \\
\star\end{array}$ & -139 & to & -127 \\
\hline MVMi & P04 & & ctca & CATGGTTGGTCAG & ttct & -107 & to & -95 \\
\hline
\end{tabular}

B.

$\begin{array}{ll}\text { B19 } & \text { P06 } \\ \text { B19 } & \text { P44 }\end{array}$

$\begin{array}{ll}\text { B19 } & \text { P06 } \\ \text { MVMi } & \text { P39 } \\ \text { B19 } & \text { P44 }\end{array}$

\begin{tabular}{|c|c|c|c|c|c|c|}
\hline 265 & & 284 & 319 & & & \\
\hline$\underset{*}{\operatorname{tattg}}$ & $\begin{array}{l}\text { GTTAGTT } \\
\star \star \star \star \star \star \star\end{array}$ & $\underset{\star}{\operatorname{ttg} t a a c g}$ & --TATAT & -73 & to & -79 \\
\hline gaact & GTTAGTT & ggggttga- & ---TATTA & -88 & to & -94 \\
\hline 2180 & & 2199 & 2249 & & & \\
\hline
\end{tabular}

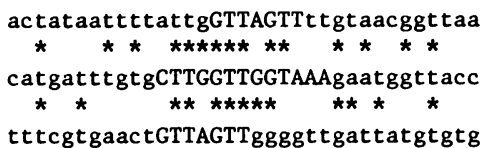

FIG. 7. Comparison of the tar element of the H-1 virus P38 promoter with the related sequence elements of the MVMi P39 and P04 promoters and B19 virus P44 and P06 promoters. Homology between two bases is indicated by an asterisk. (A) Sequences in MVM. The 13 bases of the tar elements are shown in capital letters, and their positions relative to the RNA start site are indicated on the right. The H-1 virus P04 tar is identical to the MVMi P04 tar (34). (B) Related sequence elements upstream from B19 virus promoters. The numbers above and below the sequences show nucleotide positions in the B19 viral DNA sequence. The positions (shown on the right) of the related sequences relative to the RNA start sites are estimated from the positions of the TATA boxes, since the exact start sites have not been determined. (C) Comparison of B19 virus and MVMi sequences.

H-1 and MVMi are so similar, and since a tar identical to the tar of the $\mathrm{P04}$ promoter of MVMi is present upstream of the P04 promoter of H-1 (34), we should expect the NS-1 of $\mathrm{H}-1$ virus to stimulate P04, as we have found with MVMi. Details of the H-1 virus experiment (35) were not given, although cell lines different from those in the present work were used. On the other hand, the NS protein of B19 virus was reported not to suppress transcription from the BP06 promoter (25). We suggest that, as these early promoters are relatively strong, the amount of CAT gene-containing test plasmid added to the cells may have been such that the basal level of CAT expression was already high, so the subsequent transactivation that we observed was not measurable.

Why should NS-1 activate its own promoter, while other viral regulatory proteins (for example, the $T$ antigen of simian virus 40 (32) or ICP4 of herpes simplex virus [30]), repress theirs? The reason might be that NS-1, unlike these other viral regulatory proteins, is also a component of the virus particle: NS-1 of MVM is attached to the $5^{\prime}$ end of viral DNA (13). NS-1 would be needed, towards the end of the lytic cycle, for incorporation into viral progeny.

Transcriptional promoter activity at map position 44 on the genome of B19 virus. Evidence for a transcriptional promoter activity at map position 44 on the B19 virus genome comes from the fact that the SmaI-Tth111I fragment (nt 2073 to 2277) promoted expression of the CAT gene in transfected HeLa cells. The promoter activity was lost if the viral DNA fragment was inserted in the opposite orientation. The level of CAT activity in cells transfected with pBP44-CAT was similar to that in cells transfected with pMP39-CAT. A TATA-box-related sequence (TATTAA) is found between positions 2247 and 2251 , that is about 30 base pairs upstream of the Tth111I site. CAT constructs containing restriction fragments extending further to the right (for example, to the TaqI site at position 2326 or the HindIII site at position 2430) did not promote CAT activity, possibly because the longer distance between the promoter and the CAT gene allowed initiation of translation at a wrong AUG codon. The position of the BP44 promoter relative to the beginning of the ORF of the capsid protein gene makes use of this promoter for the initiation of capsid mRNA synthesis possible. The distance between the two (positions 2250 and 2670) is about $420 \mathrm{nt}$, and in MVMi, in which the role of the MP39 promoter in the transcription of the capsid protein gene is well documented, the distance between these elements is $\mathbf{3 2 0}$ base pairs.

A feature shared by the early and late promoters of MVM and $\mathrm{H}-1$ virus is the presence of the tar sequence, about 100 base pairs upstream from the TATA boxes (discussed earlier). This is also true of the two promoters which we have detected in B19 virus (Fig. 7A): an identical sequence of $7 \mathrm{nt}$ lies upstream of the TATA box of the BP06 and BP44 promoters; this sequence occurs nowhere else in the B19 virus genome. Moreover, the sequence is homologous to the tar elements of MVM; in B19 virus, it is slightly closer to the RNA start site than the tar sequence is to the RNA start site in MVM (Fig. 7A and B). The roles that these elements in $B 19$ virus promoters may play in the regulation of viral transcription remain, as with MVM, to be demonstrated experimentally. Nevertheless, our results show that there are many similarities between the BP44 promoter and the MP39 promoter: at the structural level, they are located a similar distance upstream of the capsid protein ORF (and within the coding region of the NS-1 gene). They contain a sequence element which is also present in the promoter controlling the transcription of the nonstructural protein genes; this element resembles the tar which has been shown in $\mathrm{H}-1$ virus to play a role in the regulation of transcription by NS-1. At the functional level, both promoters have a similar activity in transfected HeLa cells. Thus, we believe that the internal promoter activity in B19 virus could be responsible for transcription of capsid protein genes, as it is in other parvoviruses.

A promoter located near the middle of the genome of B19 virus was also reported by Deiss et al. (V. Deiss, J. Tratschlin, and G. Siegl, 2nd Parvovirus Workshop Abstracts, Oxford, U.K., p. 21, 1987), who used a similar 
approach to that described in this report. However, Osawa et al. (26) proposed a transcriptional map for B19 virus in which all of the viral transcripts are initiated at the BP06 promoter. Their data were obtained by successive hybridization of filter-bound RNA from cultured bone marrow cells infected with B19 virus with several labeled viral DNA fragments. They found evidence for nine viral transcripts generated from the BP06 promoter; some transcripts had a structure unable to code for any polypeptide. Osawa et al. (26) also looked for a late promoter within the B19 virus genome by linking B19 virus DNA fragments to the CAT gene. They did not find the promoter we detected (at map position 44) because they looked only as far as map position 38.

Our results show that the BP06, but not the BP44 promoter, is transactivated by BNS-1 in nonpermissive cells. As pointed out elsewhere (27), the permissive cells represent only a minor fraction of the cells present in the bone marrow cell cultures. Thus, it is possible that the viral transcripts originating from BP06 only, as detected by Osawa et al. (26), are produced mainly during the abortive infection of the nonpermissive cells present in large excess over the permissive cells in the bone marrow culture. The uninduced activities of BP44 and MP39, although easily detectable in CAT assays, are low. We have not been able, with an RNase protection assay, to detect RNA generated from the BP44 promoter in HeLa cells transfected with $\mathrm{pEMBL} / \mathrm{B} 19$. This is not surprising, since, with the same assay, we also did not detect the mRNAs for MVM capsid proteins in the absence of transactivation of MP39 (17; data not shown).

Control of transcription in B19 virus. We have shown that transactivation by NS-1 of the promoter controlling transcription of the nonstructural protein genes occurs in B19 virus as well as in MVM. The fact that in HeLa cells the promoters BP06 and MP04 respond to BNS-1 and MNS-1 indicates that these proteins are functionally closely related and that transactivation of these promoters is not, apparently, a cell-type-specific process. The main difference we have observed in the transcriptional organization of these viruses is that in HeLa cells the capsid protein gene promoter of MVM (MP39) is strongly transactivated by MNS-1 (17), whereas the analogous promoter activity (BP44) of B19 is not stimulated by BNS-1. Perhaps there is no transactivation of the BP44 promoter by NS-1 in transfected HeLa cells because these cells lack an additional factor needed for efficient use of this promoter. Transactivation may occur in other types of cells able to support a productive infection. The tissue specificity of B19 virus may be thus related to regulation of viral transcription.

\section{ACKNOWLEDGMENTS}

We are very grateful to F. Beutler for anti-NS-1 antibody, B. Bentele for help with tissue culture, and V. Jongeneel and $J$. Rommelaere for valuable discussions. S. Cotmore and P. Tattersall kindly provided the clone pYT103.

We thank the Fonds National Suisse de la Recherche Scientifique and the University of Lausanne for financial support.

\section{LITERATURE CITED}

1. Anderson, M. J., S. E. Jones, S. P. Fisher-Hoch, E. Lewis, S. M. Hall, C. L. R. Bartlett, B. J. Cohen, P. P. Mortimer, and M. S. Pereira. 1983. Human parvovirus, the cause of erythema infectiosum (fifth disease)? Lancet i: 1378 .

2. Antonietti, J. P., R. Sahli, P. Beard, and B. Hirt. 1987. Characterization of the cell type-specific determinant in the genome of minute virus of mice. J. Virol. 62:552-557.

3. Astell, C. R., E. M. Gardiner, and P. Tattersall. 1986. DNA sequence of the lymphotropic variant of minute virus of mice, MVM(i), and comparison with the DNA sequence of the fibrotropic strain. J. Virol. 57:656-669.

4. Astell, C. R., M. Thomson, M. Merchlinsky, and D. C. Ward. 1983. The complete DNA sequence of minute virus of mice, an autonomous parvovirus. Nucleic Acids Res. 11:999-1018.

5. Berns, K. 1984. The parvoviruses. Plenum Publishing Corp., New York.

6. Blundell, M. C., C. Beard, and C. R. Astell. 1987. In vitro identification of a B19 parvovirus promoter. Virology 157: 534-538.

7. Bodnar, J. W. 1988. Sequence organization in regulatory regions of DNA of minute virus of mice. Virus Genes 2(2): $167-182$.

8. Clemens, K. E., and D. Pintel. 1988. The two transcription units of the autonomous parvovirus minute virus of mice are transcribed in a temporal order. J. Virol. 62:1448-1451.

9. Cossart, Y. E., B. Cant, A. M. Field, and D. Widdows. 1975. Parvovirus-like particles in human serum. Lancet i:72-73.

10. Cotmore, S. F., V. C. McKie, L. J. Anderson, C. R. Astell, and P. Tattersall. 1986. Identification of the major structural and nonstructural proteins encoded by human parvovirus B19 and mapping of their genes by procaryotic expression of isolated genomic fragments. J. Virol. 60:548-557.

11. Cotmore, S. F., and P. Tattersall. 1984. Characterization and molecular cloning of a human parvovirus genome. Science 226:1161-1165.

12. Cotmore, S. F., and P. Tattersall. 1986. Organization of nonstructural genes of the autonomous parvovirus minute virus of mice. J. Virol. 58:724-732.

13. Cotmore, S. F., and P. Tattersall. 1988. The NS-1 polypeptide of minute virus of mice is covalently attached to the $5^{\prime}$ termini of duplex replicative-form DNA and progeny single strands. J. Virol. 62:851-860.

14. Dente, L., G. Cesareni, and R. Cortese. 1983. pEMBL: a new family of single stranded plasmids. Nucleic Acids Res. 11: 1645-1655.

15. Dignam, J. D., R. M. Lebovitz, and R. G. Roeder. 1983. Accurate transcription initiation by RNA polymerase II in a soluble extract from isolated mammalian nuclei. Nucleic Acids Res. 11:1475-1489.

16. Doerig, C., P. Beard, and B. Hirt. 1987. A transcriptional promoter of the human parvovirus B19 active in vitro and in vivo. Virology 157:539-542.

17. Doerig, C., B. Hirt, P. Beard, and J. P. Antonietti. 1988. Minute virus of mice non-structural protein NS-1 is necessary and sufficient for transactivation of the viral P39 promoter. J. Gen. Virol. 69:2563-2573.

18. Gorman, C. M., L. F. Moffat, and B. H. Howard. 1982. Recombinant genomes which express chloramphenicol acetyltransferase in mammalian cells. Mol. Cell. Biol. 2:1044-1051.

19. Graham, F. L., and A. J. van der Eb. 1973. A new technique for the assay of infectivity of human adenovirus 5 DNA. Virology 52:456-463.

20. Jongeneel, C. V., R. Sahli, G. K. McMaster, and B. Hirt. 1986. A precise map of splice junction in the mRNAs of minute virus of mice, an autonomous parvovirus. J. Virol. 59:564-573.

21. Krieg, P. A., and D. A. Melton. 1984. Functional messenger RNAs are produced by SP6 in vitro transcription of cloned cDNAs. Nucleic Acids Res. 12:7057-7070.

22. Maniatis, T., E. F. Fritsch, and J. Sambrook. 1982. Molecular cloning: a laboratory manual. Cold Spring Harbor Laboratory, Cold Spring Harbor, N.Y.

23. Melton, D. A., P. A. Krieg, M. R. Rebagliati, T. Maniatis, K. Zinn, and M. R. Green. 1984. Efficient in vitro synthesis of biologically active RNA and RNA hybridization probes from plasmids containing a bacteriophage SP6 promoter. Nucleic Acids Res. 12:7035-7056.

24. Mortimer, P. P., R. K. Humphries, J. G. Moore, R. H. Purcell, and N. S. Young. 1983. A human parvovirus-like virus inhibits haematopoietic colony formation in vitro. Nature (London) 302:426-429.

25. Osawa, K., J. Ayub, S. Kajigaya, T. Shimada, and N. Young. 
1988. The gene encoding the nonstructural protein of B19 (human) parvovirus may be lethal in transfected cells. J. Virol. 62:2884-2889.

26. Osawa, K., J. Ayub, H. Yu-Shu, G. Kurtzman, T. Shimida, and N. Young. 1987. Novel transcription map for the B19 (human) pathogenic parvovirus. J. Virol. 61:2395-2406.

27. Osawa, K., G. Kurtzman, and N. S. Young. 1986. Replication of the B19 parvovirus in human bone marrow cell cultures. Science 233:883-886.

28. Osawa, K., and N. Young. 1987. Characterization of capsid and noncapsid proteins of $\mathrm{B} 19$ parvovirus propagated in human erythroid bone marrow cell culture. J. Virol. 61:2627-2630.

29. Pintel, D., D. Dadachanji, C. R. Astell, and D. C. Ward. 1983. The genome of minute virus of mice, an autonomous parvovirus, encodes two overlapping transcription units. Nucleic Acids Res. 11:1019-1038.

30. Preston, C. M. 1979. Control of herpes simplex virus type 1 mRNA synthesis in cells infected with wild-type virus or the temperature-sensitive mutant $t s \mathrm{~K}$. J. Virol. 29:275-284.

31. Rao, K. R., A. R. Patel, M. J. Anderson, J. Hodgson, S. E. Jones, and J. R. Pattison. 1983. Infection with parvovirus-like virus and aplastic crisis in chronic hemolytic anemia. Ann. Intern. Med. 98:930-932.

32. Reed, S. I., G. R. Stark, and J. C. Alwine. 1976. Autoregulation of simian virus $\mathbf{4 0}$ gene $A$ by $T$ antigen. Proc. Natl. Acad. Sci. USA 73:3083-3087.

33. Rhode, S. L., III. 1985. trans-Activation of parvovirus P38 promoter by the 76K noncapsid protein. J. Virol. 55:886-889.

34. Rhode, S. L., III, and P. R. Paradiso. 1983. Parvovirus genome: nucleotide sequence of $\mathrm{H}-1$ and mapping of its genes by hybridarrested translation. J. Virol. 45:173-184.

35. Rhode, S. L., III, and S. M. Richard. 1987. Characterization of the trans-activation-response element of the parvovirus H-1 P38 promoter. J. Virol. 61:2807-2815.

36. Saarinen, U. M., T. L. Chorba, P. Tattersall, N. S. Young, M. J. Anderson, E. Palmer, and P. F. Coccia. 1986. Human parvovirus B19-induced epidemic acute red cell aplasia in patients with hereditary hemolytic anemia. Blood 67:1411-1417.

37. Sahli, R., G. K. McMaster, and B. Hirt. 1985. DNA sequence comparison between two tissue-specific variants of the autonomous parvovirus, minute virus of mice. Nucleic Acids Res. 13:3617-3633.

38. Shade, R. O., M. C. Blundell, S. F. Cotmore, P. Tattersall, and C. R. Astell. 1986. Nucleotide sequence and genome organization of human parvovirus B19 isolated from the serum of a child during aplastic crisis. J. Virol. 58:921-936.

39. Siegl, G. 1976. The parvoviruses. Virology monographs 15. Springer-Verlag, Vienna.

40. Sompayrac, L. M., and K. J. Danna. 1981. Efficient infection of monkey cells with DNA of simian virus 40 . Proc. Natl. Acad. Sci. USA 78:7575-7578.

41. Struhl, K., and I. A. Hope. 1985. GCN4 protein, synthesized in vitro, binds to HIS3 regulatory sequences: implications for general control of amino acids biosynthetic genes in yeast. Cell 43:177-188.

42. Tattersall, P., and J. Bratton. 1983. Reciprocal productive and restrictive virus-cell interactions of immunosuppressive and prototype strains of minute virus of mice. J. Virol. 46:944-955.

43. Trempe, J. P., and B. J. Carter. 1988. Regulation of adenoassociated virus gene expression in 293 cells: control of mRNA abundance and translation. J. Virol. 62:68-74.

44. Ward, D. C., and P. Tattersall. 1978. Replication of mammalian parvoviruses. Cold Spring Harbor Laboratory, Cold Spring Harbor, N.Y. 